\title{
La expansión de universidades privadas en el caso argentino
}

Marisa Zelaya*

Resumen: Este trabajo aborda el tema de la privatización de la educación superior en el caso argentino. Se presentan, en primer lugar, los cambios producidos en el contexto latinoamericano y en nuestro país, en especial en el campo educativo, a partir de la instalación del paradigma neoliberal. A continuación, se describen dos momentos de expansión de universidades privadas en el caso argentino: uno es el período 1989- 1995, durante el cual crece el número de universidades privadas con sus especificidades; el segundo, abarca desde el año 1995 hasta la actualidad, y se caracteriza por la expansión del sector privado de la educación por medio de la creación de "ofertas distantes" o "Extensiones Áulicas". Por último, se aborda la dimensión espacial vinculada a la expansión de las extensiones.

Palabras clave: políticas neoliberales; privatización de la educación superior; expansión de universidades privadas; "Extensiones Áulicas".

\section{The expansion of private universities: the Argentinian case}

Abstract: This paper addresses the issue of higher education privatization in the Argentinian case. Firstly, we present the changes that have taken place in the latinoamerican context, particularly in our own educational field since the beginning of the neoliberal paradigm. In addition, two stages of the expansion of private universities in Argentina are described: one stage covers the expansion period that took place from 1989 to 1995 in which the number of private universities together with their specificities increased, and the other stage covers the period from 1995 up to now which is characterized by the expansion of private sector of education through "ofertas distantes" or "extensions extraterritoriales/ áulicas." Finally, the spatial dimension linked to the expansion of extensions are discussed.

Key words: neoliberal policies; privatization of higher education; expansion of private universities; "Extensiones Áulicas".

\section{El papel del Estado en las políticas educativas en las últimas décadas}

En el caso argentino como en la mayoría de los países latinoamericanos, el modelo de Estado de Bienestar llega a su límite en la década de los '70. Surge así en

* Universidad Nacional del Centro de la Provincia de Buenos Aires - Argentina. marisazelaya@ gmail.com 
escena la crítica a este modelo de Estado, en algunos países desarrollados ${ }^{1}$, lo que da lugar a "una revolución conservadora, que denunció en tutto el desorden económico, social e incluso moral y cultural" (Emmerich, 1996, p. 3).

La conformación del Estado (postsocial, neoliberal, neoconservador) implica un proceso de transformación del mismo que se relaciona con el inicio de las políticas de ajuste ${ }^{2}$ y estabilización y con la inserción de los países latinoamericanos ${ }^{3}$ en el marco de la política mundial.

En consecuencia, se observa que estas reformas en la mayoría de los países se fundamentan sobre el reconocimiento de la carencia de recursos del sector público para llevar a cabo las transformaciones económicas y sociales. Este proceso de transformación está asociado a un amplio espectro de políticas recomendadas por el Banco Mundial ${ }^{4}$, el Fondo Monetario Internacional y otras organizaciones financieras. Dichas políticas no sólo influyen directamente sobre el modelo económico del caso argentino para obtener así un superávit fiscal que permita cumplir los compromisos de la deuda externa, sino en otros aspectos como los culturales, políticos, sociales, etc. En este contexto la educación no ha estado ajena al desarrollo de una agenda educativa globalizada.

Por otra parte, un punto de inflexión, en particular, en la Argentina lo constituye la crisis económica, social y política del 2001-2002, de aquí en adelante se observan modificaciones significativas en torno al rol del Estado.

Autoras como Giovine, Martignoni (2010) y Senén González (2008) realizan un análisis de las actuales políticas educativas tanto en América Latina como en el caso argentino y distinguen que la primera década de los 2000 presenta un nuevo escenario educativo, ya que se está redefiniendo el papel del Estado nacional en

I Algunos de los gobiernos que respondieron a esta corriente son: el de Tatcher (Gran Bretaña, 1979), el de Reagan (Estados Unidos, 1981 ) y el de Mulroney (Canadá, 1984).

2 El ajuste estructural se vincula con dos aspectos: el primero tiene que ver con un conjunto de programas y políticas recomendadas por el BM, el FMl y otras organizaciones financieras, para que los países latinoamericanos se inserten en la economía mundial y el segundo, es el pago de la deuda externa.

3 En el caso argentino a partir del quiebre del modelo sustitutivo de importaciones y de la crisis de la deuda externa de 1982, con las consecuencias que trajo su resolución parcial (durante el gobierno del Dr. Alfonsín 1982-1989). Este proceso se refuerza con la denominada "Reforma del Estado", emprendida por el gobierno del presidente Menem ( 1989-1999) que lleva adelante el más audaz y radicalizado proceso de ajuste del aparato estatal que haya conocido la Argentina. Se produce una profunda transformación en la relación estado-sociedad y en los vínculos entre los distintos grupos, clases y actores sociales configurados durante largas décadas de nuestro país.

4 Corbalán sostiene que el Banco Mundial, además de cumplir como organismo financiero la función de otorgar préstamos, desarrolla otras funciones. Se lo puede visualizar como un complejo académico-intelectual ya que muchos de los financiamientos que el Banco Mundial otorga presuponen una cantidad de investigaciones y estudios. (Corbalán, | 998: | | 5)

Pro-Posiçôes, Campinas, v. 23, n. 2 (68), p. 179-194, maio/ago. 2012 
sentido inverso a los años 1990. Asimismo, Senén González, sitúa a este momento histórico como la etapa posneoliberal ${ }^{5}$, donde se recupera la dimensión política del discurso y del quehacer educativo.

En relación con esta etapa posneoliberal, ésta se percibe como un proceso de cambios muy complejos, no puede ser entendida sólo como la recuperación de la intervención estatal. Al respecto, se recupera el término de Ruth Felder, citada por Senén González, (2008, p. 83) quien se refiere al posneoliberalismo de la siguiente manera:

“[...] la pregunta acerca de los alcances y límites de la acción de gobiernos progresistas en América Latina no tiene una respuesta simple, porque no basta identificar las políticas que se apartan de los postulados de la economía neoclásica ni de observar el mayor o menor activismo estatal."

En función de lo expuesto, se observa que no hubo reversión de las reformas estructurales de los noventa, pero sí cambios sustanciales de la situación política. Esto se traduce en el reconocimiento de la dimensión política de las políticas públicas (repolitización de las políticas públicas), en el caso argentino y en otros países de América Latina.

Por consiguiente, se asiste a una reasunción de las obligaciones del Estado, por ende, éste se constituye en un actor clave y responsable de proveer, garantizar y supervisar la educación. Esto se ve plasmado en una serie de nuevas leyes como las siguientes: Ley de Fondo Nacional de Incentivo Docente (No 25919/04), Ley de Educación Técnica Profesional ( No 26.058/05), Ley de Financiamiento Educativo (No 26.075/04), Ley de Educación Nacional (No 26206/06), Ley de Educación Provincial (No 13688/0), entre otras.

Sin embargo, en materia legislativa el nivel superior universitario ${ }^{6}$ no ha sido objeto de modificaciones: aún se encuentra vigente la Ley de Educación Superior (No 24.521) sancionada en el año 1995. Esta ley incluye al sector privado universitario en forma paralela al sector público y define objetivos comunes para ambos sectores; a su vez, precisa mecanismos de acreditación iguales de carrera de grado y postgrado, sin distinción alguna. Por último, habilita al sector público universitario para el cobro de aranceles y la venta de servicios.

5 Sader sostiene que el Postneoliberalismo "es una categoría descriptiva que designa diferentes grados de negación del modelo, sin llegar a configurar un nuevo modelo, al mismo tiempo en que un conjunto híbrido de fuerzas compone las alianzas que están en la base de los nuevos proyectos" Sader, E. Refundar el Estado. Posneoliberalismo en América Latina, Buenos Aires, CLACSO, 2008, pag.81.

6 Chiroleu, A y lazzetta, O. sostienen que "la política universitaria se presenta como un campo en el que el gobierno de Kirchner (2003-2007) ha mostrado una falta de iniciativa y una tendencia inercial que contrasta con la audacia e innovación evidenciadas en otros rubros[...] los temas de la universidad no lograron ocupar un lugar relevante entre las preocupaciones oficiales" (2009, 32). 
El proceso de cambio de esta ley no está entre los temas prioritarios de la agenda político-educativa en la actualidad, aunque se visualizan políticas que jerarquizan la actividad científico-tecnológica y se desarrollan algunos programas focalizados en el sistema universitario como: Fortalecimiento del sistema de becas; Mejoras en la infraestructura; Becas para carreras prioritarias; entre otros.

\section{La expansión del sector universitario}

Como ya se ha mencionado, América Latina ha estado sumida en sucesivas crisis económicas durante las últimas décadas y, en particular, Argentina durante los años 2001-2002, es en este período donde, el 57,5\% de los habitantes de Argentina se encontraba por debajo de la línea de pobreza. Giovine y Martignoni (2010, p. 128) sostienen, además que la brecha de la distribución del ingreso entre el $10 \%$ más rico y el $10 \%$ más pobre de la población de la ciudad de Buenos Aires se había acrecentado de un 39,2\% en 1990 a un 89,7\% en 2000 y al 95\% en 2002, según el Instituto Nacional de Estadística y Censos (INDEC). Esto es como consecuencia de los fracasos de la mayoría de las estrategias y procesos del proyecto neoliberal.

En este marco, la tendencia de expansión, tanto pública como privada, de la educación superior argentina sigue vigente en la actualidad con nuevos parámetros de diversificación y descentralización del sistema universitario nacional. Esto es en un aparente proceso de democratización que se expresa en un ingreso con menores grados de selectividad y con escasas pautas de restricción.

En la actualidad, el sistema universitario argentino es complejo y heterogéneo, no sólo por la creciente presencia de las universidades privadas sino también por la expansión de las universidades tanto públicas como privadas a través de la modalidad de Extensiones Áulicas. En función de lo expuesto se presenta el siguiente cuadro que muestra el sistema universitario argentino, en él se visualiza tanto la acentuación del sector privado como el de las extensiones áulicas.

Cuadro $\mathrm{N}^{\circ}$ I: Universidades, institutos universitarios, extensiones áulicas nacionales, privadas y provinciales

\begin{tabular}{|c|c|c|c|c|}
\hline & Universidades & Institutos Univ. & Total & Extensiones \\
\hline Nacional & 46 & 7 & 53 & 148 \\
\hline Privada & 46 & 13 & 59 & 110 \\
\hline Provincial & 1 & - & 1 & \\
\hline Extranjeras & 1 & & 1 & \\
\hline Internacional & & 1 & I & \\
\hline Total & 94 & 21 & 115 & 258 \\
\hline
\end{tabular}

Fuente: Parte de estos datos fueron extraídos del Programa de Mejoramiento del Sistema de información Universitaria 2009. 
Este cuadro revela el crecimiento de las "Extensiones Áulicas", cabe señalar que la expansión de estas instituciones se torna significativa a partir de revalorizar la dimensión espacial y, en particular, las relaciones entre ciudades (municipios), población y universidades.

En este escenario esto estaría marcando nuevas relaciones al interior de las ciudades, pues este proceso de expansión, tiene diferencias sustantivas, en primer lugar, en relación a que cubre espacios territoriales impensados históricamente, ya sea por su reducido tamańo o por el número de su población, en la apertura de una universidad. Se constituyen ejemplos de ello las ciudades de Saladillo (29.600 hab.); Las Flores (23.551 hab.); 25 de Mayo (34.877 hab.); Bolívar (32.442 hab.) (Ver mapa No1 del anexo).

En segundo lugar, en relación a la matrícula que ingresa, ésta se compone de la población de recursos medios y bajos, que de otra manera no podría acceder a estudios superiores universitarios. En este sentido, se recuerda que en nuestro país, la brecha de la distribución de ingresos entre el nivel más alto y el más bajo es de 31 veces (INDEC, 2006).

De modo que se estaría cubriendo las necesidades educativas universitarias de una población que se ha ido empobreciendo, esto reforzaría la existencia de dos redes o circuitos de formación universitaria cada vez más diferenciadas desde las dimensiones académicas y, por ende, relacionadas con el poder adquisitivo de la población. Por consiguiente, están aquellos que pueden acceder a universidades tradicionales, consolidadas y con solvente trayectoria académica. Frente a otros que acceden a universidades privadas y a "Extensiones Áulicas", de reciente creación, aún no fortalecidas académicamente. En la mayoría de las Extensiones, se dictan carreras en infraestructuras edilicias que no se corresponde con instituciones universitarias, a su vez, con profesores que viajan cada quince días y poseen condiciones laborales precarias.

A continuación, se abordarán los procesos de expansión del sector universitario en Argentina durante las últimas décadas. Para ello se tendrán en cuenta dos momentos de expansión, que se sitúan como producto de su forma de crecimiento diferente y con características específicas.

El primer momento o período de expansión es el que corresponde al lapso comprendido entre 1989 y 1995 , en el cual se duplica la cantidad de universidades privadas. El segundo momento o período comprende desde 1995 hasta la actualidad; durante este tiempo, tanto el sector privado como el público ${ }^{7}$ de educación superior se expande a través de la creación de "ofertas distantes" o "Extensiones Áulicas".

7 Por ejemplo entre 1995-2007 se mantiene la cantidad de instituciones privadas, sólo se crean cuatro universidades estatales. Ver cuadro I. I del Anuario 2007 de Estadísticas Universitarias. S.P.U/MECT. 
En este caso, se define como "Extensión Áulica" el dictado total o parcial de una carrera de grado universitario por parte de una universidad pública o privada en una localización distante respecto de la radicación geográfica de la sede central. Asimismo, se denomina "centro universitario", "centro regional", "extensión universitaria", al lugar donde se dictan varias carreras, que pueden ser de la misma o de distintas universidades públicas y/o privadas, situación que ha provocado el crecimiento, a través de la multiplicación de carreras del sistema universitario en la Argentina. Esta se constituye en una modalidad distinta a las tradicionales formas de creación de universidades. Por lo tanto, estos procesos de expansión han producido una reconfiguración de los sistemas en su conjunto y, a su vez, en las mismas instituciones del sector educativo.

En función de lo expuesto, se infiere que el proceso de expansión, tiene como principal característica un crecimiento acelerado en poco tiempo pero sin ningún tipo de planificación, lo que ha producido una oferta que "se puede caracterizar como una superposición fragmentada e ineficiente orientada en la última década por el perfil potencial consumidor" (Dirié, C y otros, 2002, 8).

Seguidamente, analizaremos con más detenimiento, cada uno de los momentos anteriormente esbozados.

\section{Primer período ( 1989 - 1995): la expansión de universidades privadas}

En este apartado se hace hincapié especialmente en la expansión de las universidades privadas -no elitistas- durante el período 1989 a 1995 en la Argentina.

El crecimiento de las universidades privadas, en la mayoría de los países de América Latina se produce a fines de la década de 1970, pero en Argentina en especial se desarrolla a principios de 1990. Estas universidades privadas tienden a ocupar un espacio no cubierto hasta ese momento por el sector público ni por el sector privado, si bien ya se habían desarrollado las universidades privadas católicas y las que están en contacto con el sector empresarial y el mercado de trabajo, denominadas seculares de elite.

Esta nueva expansión está vinculada tanto a las carreras profesionales tradicionales como a campos de estudios más recientes y novedosos. Las instituciones se orientan más hacia el mercado de trabajo y ofrecen carreras de corta duración, de esta manera parecen responder más rápido a los variados cambios del mercado.

En este caso podemos citar lo que sostiene Levy, cuando refiere que las universidades privadas no elitistas crecen por ciertas causas distintivas, pero sobre todo porque dicho sector no elitista no los excluye.

En este período 1989-1995 se crearon 20 universidades privadas frente a 9 públicas. Por consiguiente, autores como Balan y García de Fanelli sostienen que 
"la dinámica de la oferta del sector privado en Argentina se vincula estrechamente con las políticas encaradas por el Estado en relación con el sistema de educación superior" (1997, p. 23)

A continuación, se establecen algunas características propias de la oferta del sector privado de educación superior, que comprenden: financiamiento, áreas de conocimiento, tamaño de las instituciones, matrícula, ubicación geográfica.

Las universidades privadas en nuestro país tienen una gran dependencia financiera, de las cuotas que pagan los estudiantes y, en algunos casos, de las donaciones que reciben del sector privado. En este sentido, Balan y García de Fanelli sostienen que las universidades propenden a reducir costos concentrándose en áreas de conocimiento en las que no sólo la demanda sea significativa, sino también los gastos en equipamiento y materiales de enseńanza sean bajos. (1997, p. 67).

En consecuencia, se establece lo siguiente con respecto a las carreras: 1-) las áreas de conocimiento más baratas involucran a las Ciencias Sociales: Psicología, Derecho, Pedagogía, también incluyen las carreras de Administración, Ciencias Económicas y Organización y carreras del área de Ciencias de la Comunicación, Turismo y Trabajo Social; 2-) en el campo tecnológico se encuentran las carreras más orientadas hacia la Informática que las vinculadas a la Ingeniería; 3-) crecimiento de nuevas ofertas de universidades privadas con cursos de posgrado en forma simultánea con el lanzamiento de sus carreras de grado; 4-) incorporación de carreras del área de Medicina (hay que considerar que estas últimas aprovecharon la infraestructura de los centros de investigación y realizaron convenios con hospitales y centros de salud, sin invertir grandes sumas de dinero).

En resumidas cuentas, podemos observar un crecimiento importante en varias áreas de dichas universidades, pero sobre todo la de Ciencias Sociales.

Para una mejor comprensión debemos tener en cuenta cómo se agrupan las universidades de acuerdo al tamaño de las mismas. Marta Kisilevsky en su trabajo de Estadísticas Básicas de Universidades Privadas, las clasifica siguiendo un orden decreciente en función de la cantidad de alumnos matriculados en el año 1994. Podemos mencionar tres grupos: 1) Universidades Grandes: con más de 5000 alumnos, 2) Universidades Medianas: entre 4999 y 2000 alumnos, 3) Universidades Pequeñas: con menos de 2000 alumnos.

La autora mencionada sostiene que las universidades grandes registraron un crecimiento anual igual al promedio descripto. En el caso de las universidades medianas, la tasa de crecimiento fue inferior a la media de un 5,1\%. Y, por último, las universidades pequeñas son las que registraron la mayor tasa de crecimiento: un $10 \%$. Es importante distinguir que 13 de las 19 universidades pequeñas fueron creadas a partir de 1990, lo cual incide en este crecimiento. Al respecto, opina Kisilevsky que: 
El análisis de la composición porcentual indica que las universidades grandes concentraron en ambos extremos del período el mismo volumen de estudiantes (62\%), las medianas disminuyeron su participación (de 29\% al 26\%) mientras que las pequeñas lo aumentaron (del $9 \%$ al 12\%) (Kisilevsky, M. y otros 1995, p. 7)

Cabe destacar como dato importante que las 21 universidades creadas antes de 1990 disminuyeron su participación relativa al 90,9\% en el año 1994. En cambio las universidades creadas a partir de ese año fueron captando un porcentaje significativo de la población estudiantil dado que pasaron de una participación en el total de la matrícula, del $6 \%$ en el ańo 1992, al 9\% en 1994 y al 10,2 \% en 2003.

En relación a las carreras de pregrado y grado del sector privado, se destaca, en primer lugar, que la demanda por enseñanza privada aumentó considerablemente en el período 1995 hasta la actualidad si se tiene en cuenta que la tasa de promedio de crecimiento anual de los estudiantes ha sido del 6,4\%; de los nuevos inscriptos el 5,6\% y de egresados el 7,9\%.

En esta primera fase de desarrollo la mayoría de estas universidades se encuentra en Buenos Aires, (33 instituciones) y en los partidos del Gran Buenos Aires (Ver mapa No 2 del anexo), es decir, se concentra el $47 \%$ del sector privado en esta zona.

Por otra parte, Garcia de Fanelli (1997) sostiene que podemos distinguir tres tipos de instituciones con características particulares: las universidades católicas, las de orientación empresarial (no elitistas) y aquellas con pretensiones de alto nivel académico.

En síntesis, en este primer momento se establece que la creación de universidades no elitistas que se corresponde con el período histórico 1989-1995, surge como una reacción al sector público, pero también al sector privado existente. De modo que, entre las universidades privadas se diferencian dos circuitos: uno elitista y otro ligado a grupos de población de sectores medios y bajos.

Segundo período (desde 1995 a la actualidad): la expansión de las "Extensiones Áulicas"

La tendencia de expansión tanto pública como privada de la educación superior argentina sigue vigente en la actualidad con nuevas modalidades o nuevas formas de enseñanza, como son las "Extensiones Áulicas".

Cabe explicitar que distintos autores como Marano (2006), Pérez Rasetti (2008), Suasnábar y Rovelli (2009) sostienen que los términos como "sedes", "Extensiones Áulicas", "ofertas distantes" y otras derivaciones se consideran sinónimos, ya que significan la réplica de carreras en una localización distinta de la casa central donde está la universidad. 
Se ha sostenido que entre los años 1989 hasta 1995, las universidades públicas han crecido en 9 instituciones, mientras que en el sector privado el crecimiento significó un aumento de 20 instituciones; sin embargo, el número de "Extensiones Áulicas" se eleva a más de 258 desde 1995 a la actualidad.

A continuación se sintetizarán algunas características propias de este tipo de expansión como: la falta de planificación; expansión ficticia de títulos; las ofertas; las demandas; las condiciones de trabajo docente; las modalidades de conquista de los espacios territoriales y el "vacío normativo e informativo".

En primer lugar, en relación a la falta de planificación, Krotsch y García de Fanelli ${ }^{8}$ (2006) sostienen que esto implica un riesgo con respecto a la expansión territorial, ya que no se visualiza ningún tipo de combinación interinstitucional entre las instituciones de educación superior dentro de un mismo territorio, más de una vez se crean carreras sin una adecuada planificación, tanto dentro de su institución como en concordancia con la oferta ya existente en el ámbito regional. Esto pone en evidencia, como uno de los aspectos más significativos de esta expansión, la creciente competencia y a su vez la creciente heterogeneidad institucional.

En este segundo período emerge un sistema universitario muy complejo, ya que las instituciones que redefinen sus misiones buscan diferenciarse unas de otras en la búsqueda de nichos sociales y regionales en las que realizar sus actividades, es decir en establecer "Extensiones Áulicas" (Krotsch, 2006). Esto lleva a reflexionar acerca de una consecuencia indirecta de lo anteriormente expuesto que es la disputa que se desata en torno al control del espacio territorial.

A la problemática de la falta de planificación se le añade la expansión ficticia de los títulos. Uno de los temas interrelacionados con la expansión de esta modalidad es la creación muy amplia de títulos, aunque similares en cuanto a los planes de estudios existentes. Krotsch (2006) señala la incongruencia entre la enorme expansión de los títulos en términos de diversidad de nombres pero resalta que esta expansión de títulos no es tal ya que los mismos poseen contenidos curriculares y planes de estudios similares. Un ejemplo de esto es lo que sucede en el CPRES Metropolitano ${ }^{9}$ en donde existen aproximadamente treinta y tres universidades, entre públicas y privadas, y los títulos que se cuantifican son entre mil ochocientos

8 Seminario Inter-CPRES "Las localizaciones regionales, sedes, subsedes, "Extensiones Áulicas", centros distantes: La evaluación de su calidad y pertinencia”. 4-5 de octubre 2006- CPRESSecretaría de Políticas Universitarias-MECyT.

9 CPRES- Metropolitano: Ámbito geográfico Ciudad de Bs.As., Provincia de Bs. As: Partidos de Almirante Brown, Avellaneda, Berazategui, Estaban Echeverría, Ezeiza, Florencio Varela, General San Martín, General Sarmiento, Hurlingham, Ituizangó, José C. Paz, La Matanza; Lanús, Lomas de Zamora, Malvinas Argentinas, Merlo, Moreno, Morón, Quilmes, San Fernando, San Isidro, Tigre, Tres de Febrero, Vicente López. 
o dos mil, siendo de cuatro mil en el sistema universitario en su conjunto. El autor afirma que uno de los conflictos que trae aparejada la expansión de las "Extensiones Áulicas" es el "problema de falsos títulos y ofertas similares". Sostiene que la expansión da lugar a un sistema espurio o de diversificación formal, cuando estas diferenciaciones aparentes, de falsos títulos y sobreposiciones, no tiene que ver con una complejización del conocimiento sino, básicamente, con un posicionamiento frente a la lógica del mercado.

En su mayoría, estas ofertas ${ }^{10}$, llamadas de "tiza y pizarrón”, y del área de Ciencias Sociales, se expresan en duplicaciones de las mismas en espacios cercanos, en una lógica que Krostch denomina simétrica. En este caso, la competencia está más orientada a la duplicación, como mecanismo defensivo de las instituciones, que a la diferenciación institucional. Y cuando esto se observa, se trata de aspectos más bien formales del título, que de contenidos sustantivos. Esto estaría contribuyendo a un "proceso de desagregación", de la universidad y de la institución de origen, que no se puede vincular a un proceso de descentralización, por lo menos en los aspectos positivos que se pretenden con dicho proceso.

Otra característica es la que se refiere a la oferta de estas modalidades. La matrícula tiene una importancia fundamental para las instituciones de educación superior, porque por un lado posibilita el cobro de aranceles y/o en segundo lugar permite negociar mayor presupuesto con el Estado, para la universidad sede. Se considera que estos son algunos de los motivos que están en la base de la expansión.

Con respecto a la demanda se señala que operan factores llamados "socios económicos y políticos" que traccionan y facilitan esta expansión. Entre los primeros factores, podemos mencionar -sobre todo en ciudades intermedias o pequeñas ${ }^{11}$ - una demanda de la población socioeconómica de estratos medios y bajos, potencialmente insatisfecha, que por distintos motivos (costo económico, cultural, psicológico, etario, laboral) no pueden movilizarse hacia las universidades o unidades académicas centrales. Sin embargo, esta población paga cuotas para acceder a este tipo de estudios. A su vez, en estas ciudades, la sociedad civil local impulsa estas implantaciones territoriales. Esto supone un movimiento de recursos materiales pero también simbólicos, lo que a su vez incide como reclamo en el escenario político local, alentando la demanda por la vía del campo político.

En lo que se refiere a las condiciones del trabajo docente, en estas instituciones los profesores viajan, generalmente cada quince días. Además desempeñan fun-

10 Este fenómeno de expansión se ha dado en llamar, en otros países, universidades "garage" o "patito", que son formas espurias de la oferta universitaria, con algunas características similares a lo que ocurre en nuestro país. En Brasil, este tipo de universidades se denominan "polos".

II Se considera a las ciudades intermedias, las que poseen entre 10.000 y 250.000 habitantes y pequeñas a las que poseen menos de 10000 hab. Este criterio de tamaño de ciudades es tomado de Susana Aparicio (2005). 
ciones, independientes al carácter de su designación en la universidad de origen o sede, que pueden ser ad-honorem o rentadas.

Por consiguiente, estas Extensiones se constituyen en un trabajo extra, acontece como otra fuente de ingreso en relación al trabajo de la universidad sede, por ende, se percibe un sobresueldo. Para Marano (2006, p. 119), estas instituciones representan una ampliación de sus mercados de trabajo. La tarea en estas instituciones resulta especialmente un beneficio económico porque más allá de los vínculos con los alumnos, no existen otros enlaces académicos que retengan al docente como ocurre en la sede central (participación en grupos de investigación, redes académicas, publicaciones, etc.). Este beneficio económico representa un engrosamiento de los haberes para todos los cargos docentes. En estas instituciones no se contempla, al momento del contrato, la formación académica de postgrado. Además, con relación a la carrera académica y a la política salarial se establece una homologación salarial para el conjunto de docentes, sin contemplar en el salario la antigüedad, como otros indicadores de desempeño académico y profesional, sólo se contempla la situación de revista titular, jefe de trabajos prácticos y ayudantes. De modo que, estas condiciones suman a la precarización laboral de los docentes.

\section{Modalidades de conquista de los espacios territoriales: las "Extensiones Áulicas"}

La ubicación de "Extensiones Áulicas" de la década de los noventa, va marcando una cambiante configuración/ reconfiguración del sistema superior universitario. Las transformaciones acontecidas desde hace más de dos décadas llevan a pensar e interrogarnos acerca de la organización espacial de los sistemas de educación superior en forma diferente. En este caso, las "Extensiones Áulicas": a-) pueden haber sido creadas para responder a la explosión de la demanda y, en parte, fueron distribuidas funcionalmente en espacios regionales compartidos; b-) pueden haber sido creadas en lugares donde se privilegian otras lógicas, como las políticas y las económicas; c-) esta reorganización/ reconfiguración puede haber traído una complementariedad entre las instituciones de educación superior universitaria o conflictos entre los municipios, para ganar la ubicación de una institución universitaria, entre otros.

Esta expansión podría resultar, para las universidades tanto públicas como privadas, una forma de conquistar espacios territoriales con el objeto de captar matrícula y nuevas formas de obtener ingresos. Pero a su vez, esto estaría marcando nuevas relaciones al interior de las ciudades donde se instalan estas instituciones, ya que éstas se transforman en la única oportunidad de la población de recursos medios y bajos de acceder a estudios universitarias. De modo que, a este tipo de instituciones accede una población estudiantil heterogénea, con características 
distintivas, y la mayoría de los estudiantes entran a la carrera con sobre edad, es decir una edad superior a la que lo hacen habitualmente los que ingresan a otras universidades tradicionales.

En la actualidad se está frente a un escenario de "vacío normativo, estadístico e informativo", acerca de las "Extensiones Áulicas". Esto significa que la mayoría de las nuevas ofertas que las universidades han instalado en otras localizaciones a través de esta modalidad se implementaron sin evaluación, no cuentan con un reconocimiento oficial específico ${ }^{12}$, por ende no han sido registradas como tales en el sistema de estadísticas universitarias, es decir, no figuran en ningún registro oficial y esto hace a la falta de información para fines comparativos.

Sin embargo, un aporte importante lo realizan los autores Del Bello, Barsky y Giménez (2007, p. 305) quienes sostienen que el $86 \%$ de las sedes, rectorados, extensiones áulicas, y/o unidades regionales se localiza en la Ciudad de Buenos Aires, en los 24 partidos del Gran Buenos Aires y el resto en la provincia de Buenos Aires. Asimismo las restantes extensiones se distribuyen en las provincias del país, tal como se demuestra en el siguiente cuadro:

Cuadro No 2: Extensiones Áulicas en diversos espacios geográficos.

\begin{tabular}{|c|c|c|c|}
\hline Espacios Geográficos & $\begin{array}{c}\text { Extensiones Áulicas } \\
\text { Sector Estatal }\end{array}$ & $\begin{array}{c}\text { Extensiones Áulicas } \\
\text { Sector Privado }\end{array}$ & Total \\
\hline Ciudad de Buenos Aires & 11 & 33 & 44 \\
\hline $\begin{array}{c}\text { Gran Bs. As. ( } 24 \\
\text { partidos) }\end{array}$ & 22 & 15 & 37 \\
\hline Provincia de Buenos Aires & 28 & 14 & 42 \\
\hline Provincias del país & 87 & 48 & 135 \\
\hline Total General & 148 & 110 & 258 \\
\hline
\end{tabular}

Fuente: Datos Extraídos de: Del Bello, Barsky y Giménez (2007:305) "La Universidad Privada Argentina".

En función de lo expuesto anteriormente, se observa esta expansión y superposición, en el dictado de carreras de Abogacía en el radio geográfico de la zona del sudoeste de la Provincia de Buenos Aires (Tradicionalmente zona de influencia de la Universidad Nacional Centro Provincia Buenos Aires, como se visualiza en el mapa No1 del anexo. En la ciudad de Las Flores se duplica la oferta del dictado de la carrera mencionada, por un lado depende de la Universidad Nacional de Lomas de Zamora (UNLZ) y por otro, sólo a unos pocos metros de recorrido, se dicta con modalidad a distancia dependiente de la Universidad Católica de Salta (UCASAL). A su vez, a $80 \mathrm{Km}$, en Azul, se dicta esta carrera, dependiente de la

I2 Si bien durante esta época se encuentran los Decretos I 047/99 y la Resolución I I 80 /07 con el fin de que las universidades presenten información acerca de sus extensiones, la mayoría no han respetado los recaudos que prevé este marco regulatorio.

Pro-Posições, Campinas, v. 23, n. 2 (68), p. 179-194, maio/ago. 2012 
Universidad Nacional del Centro de la Provincia de Buenos Aires (UNCPBA); a $170 \mathrm{Km}$, en Tandil como extensión de la Universidad de la Fraternidad de Agrupaciones Santo Tomas de Aquino (FASTA); a 110 Km, en Olavarría, como extensión de la Universidad Nacional de Lomas de Zamora (UNLZ), a $220 \mathrm{~km}$ en Mar del Plata, se dicta la carrera, de la Universidad Nacional de Mar del Plata (UNMDP) y un poco más alejado, en la ciudad de Dolores se dicta Abogacía, dependiente de la Universidad Atlántida Argentina (UAA). En síntesis, entre un radio geográfico de 80 a 220 kilómetros, se dicta la misma carrera en 6 ciudades diferentes. Otros casos paradigmáticos de universidades privadas, son los de la Universidad Católica de Salta (UCASAL) y de Santiago del Estero quienes poseen localizaciones diseminadas en todo el país.

Frente a esta situación, en particular en la Provincia de Buenos Aires, la información acerca de la cantidad de "Extensiones Áulicas", de carreras, de alumnos y de docentes, continúa siendo una incógnita. La falta de datos sistemáticos por parte de la Secretaria de Políticas Universitarias, se confirma en este punto como uno de los más importantes.

En síntesis, este fenómeno está induciendo a un nuevo mapa universitario, lo que implica cambios sustanciales en los procesos de expansión, diversificación, y segmentación del sistema de educación superior universitario. Por eso, muchas instituciones participan de un movimiento para alcanzar nuevos mercados a través de un acercamiento a las localidades, el empleo de la educación a distancia, y últimamente, la flexibilidad de los trámites de revalidación de estudios terciarios parciales o finalizados. Todo lo expuesto estaría coadyuvando a acentuar circuitos diferenciados de formación universitaria, en relación a la población destinataria.

Con respecto al contexto argentino, Marano argumenta (2006, p. 115) que las "Extensiones Áulicas", "aunque no declaradas ni aceptadas como tal, actúan como una fuente externa o fuente privada de financiamiento. En realidad, esta diversificación del financiamiento oscurece la relación público-privado”.

En función de lo expuesto, Brunner (2006) señala que la mercantilización es más amplia que la simple promoción de nuevas instituciones privadas, y supone también otros mecanismos, como la introducción de la lógica del mercado en la gestión y gobierno de las instituciones públicas, la orientación de los "productos" hacia el mercado, el gerencialismo y la venta de servicios para la obtención de recursos financieros. Estamos aquí pues, nuevamente, frente a un proceso multidimensional -que combina diversas dimensiones de los sistemas y de las instituciones- y que ocurre a lo largo de un continuo que corre entre el extremo de lo totalmente público hasta el extremo opuesto de lo absolutamente privado, en un escenario desierto de información. 


\section{Referencias bibliográficas}

AGUILAR VILLANUEVA, L. F. El futuro del Estado-Nación. México: Visión Crítica de la Globalidad, 1998. Centro Latinoamericano de la Globalidad.

ALTBACH, P. G. Funciones complejas de las universidades en la era de la globalización. En: La educación superior en el mundo. Global University Network for Innovation, 2007.

BALAN, J.; GARCÍA DE FANELLI, A. M. El sector privado de la educación superior. En: ROLLIN KENT (Comp.). Los temas críticos de la educación superior en América Latina. México: Fondo de Cultura Económica, 1997. v. 2.

DEL BELLO, J. C.; BARSKY, O; GIMÉNEZ, G. La universidad privada argentina. Buenos Aires: Libros del Zorzal, 2007.

BRUNNER, J. Mercados universitarios: ideas, instrumentaciones y seis tesis en conclusión. Santiago de Chile, 2006. Mimeo.

CLARK, B. El sistema de educación superior. Una visión comparativa de la organización académica. México : Universidad Autónoma Metropolitana, 1993.

CORBALÁN, A. Banco Mundial: consideraciones acerca de sus estrategias políticas. Revista Educación, Actualidad e Incertidumbre. Tandil: NEES- FCH- U.N.C.P.B.A, 1998.

DIDOU AUPETIT, S. Ciudad y universidad. Dinámica de organización en México, 19701994. Mexico: Biblioteca de la Educación Superior, 1998. Serie Ensayos.

DIDOU AUPETIT, S. La transnacionalización de la educación superior, aseguramiento de la calidad y acreditación en México. México: DIE-CINVESTAU, 2002.EMMERICH, G. E. América Latina: del Estado Productor/Benefactor al Estado Controlador/Impulsor. En: CONGRESO INTERNACIONAL: ESTADO-SOCIEDAD: LAS NUEVAS REGLAS DEL JUEGO, 1996. p. 1-16.

GARCÍA DE FANELLI, A. M. La expansión de las universidades privadas. Revista Pensamiento Universitario, Buenos Aires, año 5, n. 6, p. 56-72, 1997.

GARCIA DE FANELLI, A. M. La educación transnacional: la experiencia extranjera y lecciones para el diseño de una política de regulación en la Argentina. Buenos Aires: Documento de la CONEAU, 1999. p. 1-36.

GARCÍA DE FANELLI, A. Las localizaciones regionales, sedes, subsedes, extensiones áulicas, centros distantes: la evaluación de su calidad y pertinencia. En: SEMINARIO

INTER-CPRES, 1.2 y 3 de oct. 2006. Acta n. 236. Secretaría de Políticas UniversitariasMECyT. Disponible en: http://www.me.gov.ar/spu/guia_tematica/CPRES. Aceso en: oct. 2006.

GARCÍA DELGADO, D. Estado y sociedad. La nueva relación a partir del cambio estructural. Buenos Aires: FLASCO; Tesis Norma, 1994.

GARCÍA GUADILLA, C. Conocimiento, educación superior y sociedad en América Latina. Caracas: Centro de Estudios del Desarrollo. Nueva Sociedad, 1996. 
GIOVINE, R.; MARTIGNONI, L. Politicas educativas e instituciones escolares en Argentina. Tandil: UNCPBA-Secretaría Académica, 2010.

KISILEVSKY, M. et al. Estadística básica de universidades privadas. Años 1984-1994. Buenos Aires: Secretaría de Políticas Universitarias; MCE, 1997. p. 1-54.

KROTSCH, P. Las localizaciones regionales, sedes, subsedes, extensiones aulicas, centros distantes: la evaluación de su calidad y pertinencia. En: SEMINARIO INTER-CPRES, 1. 2 y 3 de oct. 2006. Acta n. 236. Secretaría de Políticas Universitarias-MECyT. Disponible en: http://www.me.gov.ar/spu/guia_tematica/CPRES. Aceso en: oct. 2006.

LENS, J.L. Los proyectos educativos de las agencias multilaterales y su influencia en los sistemas educativos de la región de América Latina. Revista Educación, Actualidad e Incertidumbre. Tandil: NEES- FCH- U.N.C.P.B.A, p.54-78, 1998.

LEVY, D. La educación superior y el Estado en Latinoamérica. Desafios privados al predominio público. México: Porrúa, 1997.

PÉREZ LINDO, A. Prologo. En: ALTBACH, P. G; MCGILL PETERSON, P. Educación superior en el Siglo XXI. Desafio global y respuesta nacional. Buenos Aires: Biblos, 2000. p. 3-7.

SADER, E. Refundar el Estado. Posneoliberalismo en América Latina. Buenos Aires: CLACSO, 2008. p. 81.

SENÉN GONZÁLEZ; NOVICK, S. Política, leyes y educación. Entre la regulación y los desafíos de la macro y micropolítica. En: PERAZZA, R. (Comp.). Pensar lo público. Buenos Aires: Aique Grupo, 2008.

SOUSA SANTOS, B. de. La universidad del siglo XXI. Para una reforma democrática y emancipadora de la universidad. Buenos Aires: Miño y Dávila, 2005.

SUASNAVAR, C.; ROVELLI, L. Mapeo del sistema de educación superior en el área metropolitana de Buenos Aires. Algunas consideraciones sobre su expansión y coordinación. Buenos Aires: Observatorio Sindical de Políticas Universitarias del Instituto de Estudios y Capacitación de la CONADU. En: ENCUENTRO NACIONAL, 6., Y LATINOAMERICANO, 3., "LA UNIVERSIDAD COMO OBJETO DE INVESTIGACIÓN", Cordoba. Anais... Argentina, Universidad Nacional de Córdoba, p. 1-20, 2009.

Recebido em 19 de julho de 2011 e aprovado em 04 de novembro de 2011. 


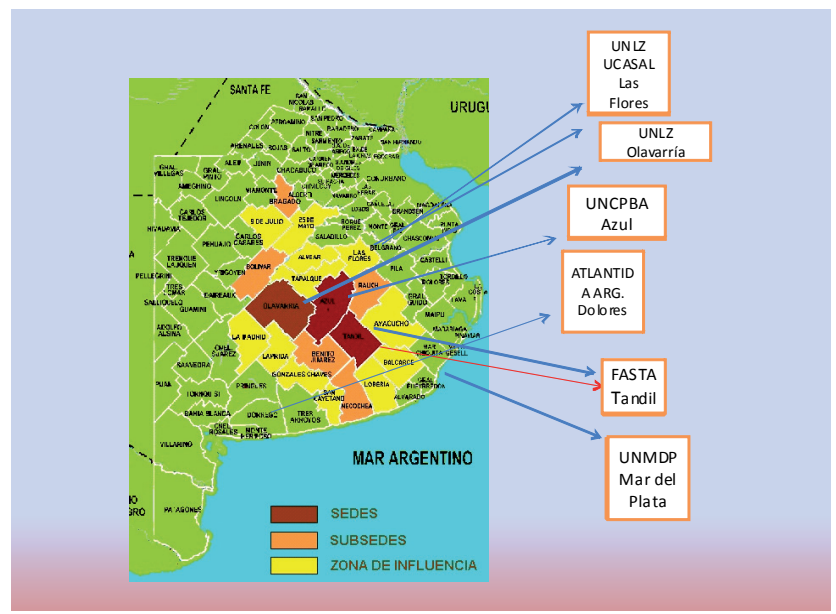

Figura I. Expansión y superposición de carreras de Abogacía en el radio geográfico del sudoeste de la provincia de Buenos Aires. Zona de influencia de la UNCPBA (período 1995-2010).

Fuente: Elaboración propia

Referencias: Universidad Nacional de Lomas de Zamora (UNLZ). Universidad Católica de Salta (UCASAL), Universidad Nacional del Centro de la Provincia de Buenos Aires (UNCPBA), Universidad Atlántida Argentina (UAA), Universidad de la Fraternidad de Agrupaciones Santo Tomas de Aquino (FASTA), Universidad Nacional de Mar del Plata (UNMDP)

Figura 2.

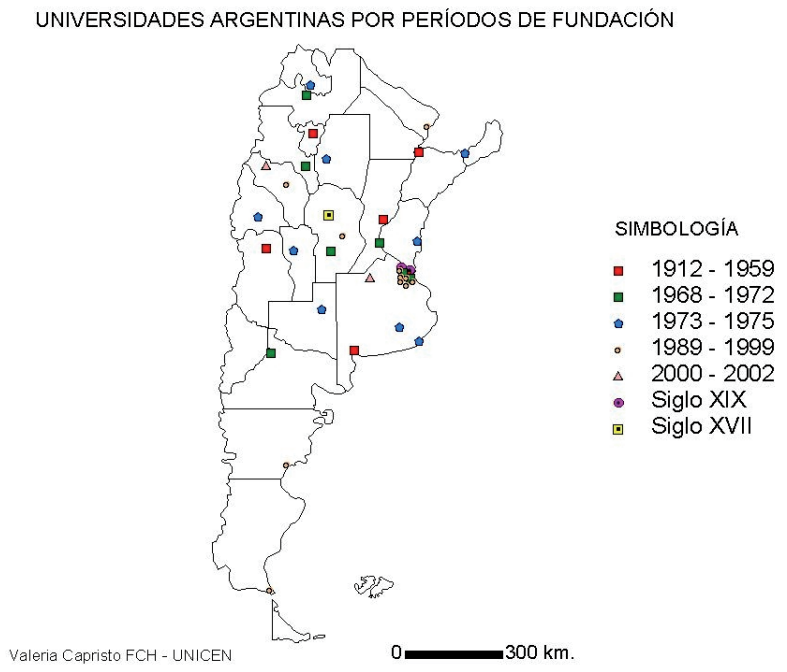

